\title{
A Study of Wireless Sensor Network Routing Protocols for Maintenance Access Hatch Condition Surveillance
}

\author{
Hoo-Rock Lee ${ }^{\star}$, Kyung-Yul Chung ${ }^{*}$ and Kyoung-Son Jhang ${ }^{* *}$
}

\begin{abstract}
Maintenance Access Hatches are used to ensure urban safety and aesthetics while facilitating the management of power lines, telecommunication lines, and gas pipes. Such facilities necessitate affordable and effective surveillance. In this paper, we propose a FiCHS (Fixed Cluster head centralized Hierarchical Static clustering) routing protocol that is suitable for underground maintenance hatches using WSN (Wireless Sensor Network) technology. FiCHS is compared with three other protocols, LEACH, LEACH-C, and a simplified LEACH, based on an ns-2 simulation. FiCHS was observed to exhibit the highest levels of power and data transfer efficiency.
\end{abstract}

Keywords - Maintenance Hatch, Underground Facilities, WSN, Routing Protocol, ns-2

\section{INTRODUCTION}

A low-cost, high-efficiency surveillance method is critical for engineers monitoring the conditions or states of target locations. The WSN technology that is currently actively under study may be the most appropriate to implement such needs. WSN can particularly be applied to status surveillance in power supply lines to detect symptoms or signs of power line problems beforehand. However, to practically implement WSN we first need to consider the geographical placement of maintenance access hatches through which we can access underground power lines, as shown in Fig. 1.

Sensors monitoring underground power lines should be located within such access hatches. The sensors can send status data to a base station or an electric power substation. Fig. 1 shows an electric power substation, including a large distribution of maintenance access hatches in a mesh-like topology, which is particularly common in urban areas. Sensor nodes placed within maintenance hatches can communicate with wireless relay transmitters on the ground, but cannot communicate with other sensor nodes in access hatches. The relay node on the ground can transmit and receive between relay nodes, but cannot communicate over radio signal coverage.

\footnotetext{
* This work (NO. 2011-515) was supported by the Energy Information Technology Development and Energy Policy Support Program of the Electric Power Public Tasks Evaluation \& Planning Center (ETEP) grant, which is funded by the Korean government's Ministry of Knowledge Economy

Manuscript received May 11, 2012; first revision July 4, 2012; accepted October 25, 2012.

Corresponding Author: Kyoung-Son Jhang

* Korea Institute of Machinery and Materials, 156, Gajeongbuk-Ro, Yuseonggu, Daejeon, Korea (\{hrock, kychung\} @kimm.re.kr)

** Dept. of Computer Engineering, ChungNam National Univ., 220, Kung-Dong, Yusong-Gu, Daejeon, 305-764, Korea (sun@cnu.ac.kr)
} 


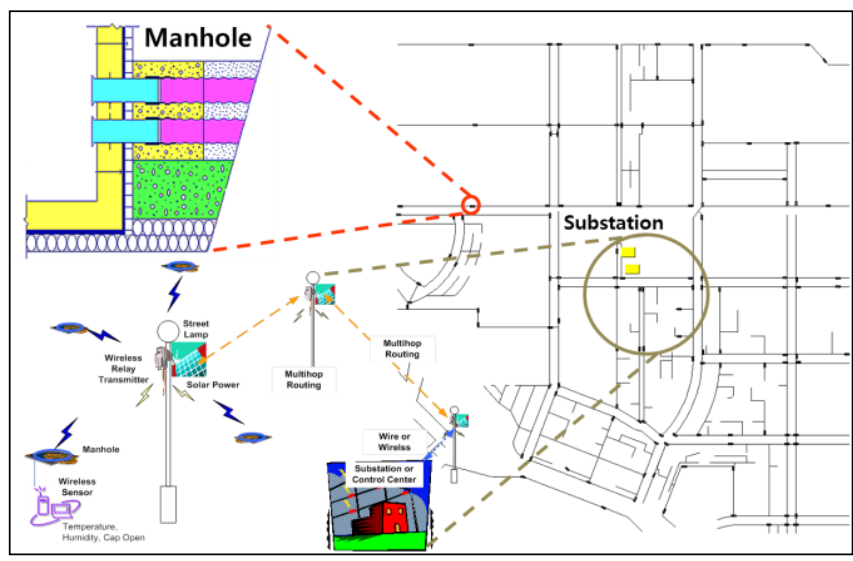

Fig. 1. WSN diagram for urban underground power line manhole surveillance

The relay nodes have a solar power module and adequate sized battery for an operational lifetime.

In this paper, we assume that a hierarchical WSN is suitable for the internal state monitoring of all maintenance access hatches in a substation. We thus evaluate several hierarchical routing algorithms (LEACH, LEACH-C, Static Clustering [1] and the proposed algorithm) based on an ns-2 network simulator with settings similar to the environmental conditions of actual facilities for a city's underground power lines. This paper is organized as follows: Section 2 describes related work, followed by a detailed description of our proposed routing algorithm in Section 3. Performance comparisons with other hierarchical routing algorithms are presented in Section 4. Section 5 details the conclusions.

\section{RELATED WORK}

LEACH is a hierarchical routing algorithm for sensor networks. It is aimed at making energy consumption in each node uniform by selecting $\mathrm{CH}$ (Cluster-Heads) for the next round based on the function $P_{i}(t)$, which indicates the possibility to become the next $\mathrm{CH}$. The function $P_{i}(t)$ is calculated in each node of a cluster at the start of each round. This function is also selected in such a way that the expected number of $\mathrm{CH}$ nodes for a round remains a constant $\mathrm{k}$ [2].

$$
\mathrm{E}[\# \mathrm{CH}]=\sum_{\mathrm{i}=1}^{\mathrm{N}} \mathrm{P}_{\mathrm{i}}(\mathrm{t}) \times 1=\mathrm{k}
$$

LEACH-C is a modified version of LEACH, where each node sends information about its current location and energy level to the base station. The base station runs an optimization algorithm to determine the clusters for that round and sends the results to each node. Each node should have GPS information to which it will send its location information.

Static-Clustering is a protocol based on LEACH-C, which determines the $\mathrm{CH}$ in the first round and does not change the $\mathrm{CH}$ any further, as the name indicates. 
In a recent study on the optimal number of cluster heads for $\mathrm{LEACH}$, another calculation method was proposed [3]. It assumes that the area of each cluster is a circle of a given radius and that the cluster head located at the center of the cluster has its own respective node distribution density. The paper [3] exhibits an inter-relationship among the optimal number of cluster heads, the number of data frames and the distance between cluster heads and the base station.

Regarding the study based on wireless underground sensor networks, several studies employing various topology, data aggregation and routing methods have been proposed for the monitoring of soil conditions and the monitoring of the interior of mines by installing wireless sensors under and on the ground [4-6].

\section{Proposed Protocol, for a Fixed Cluster Head}

To facilitate hierarchical routing, we assume that the $\mathrm{CH}$ is placed in such a way so as to ensure easy relay of surveillance data from the nodes in a cluster and that it has more power than the usual nodes in maintenance hatches.

We first devised an algorithm called a 'simplified LEACH', based on LEACH, but without a cluster head determination process since cluster heads are fixed. Fig. 2 shows the set-up phase

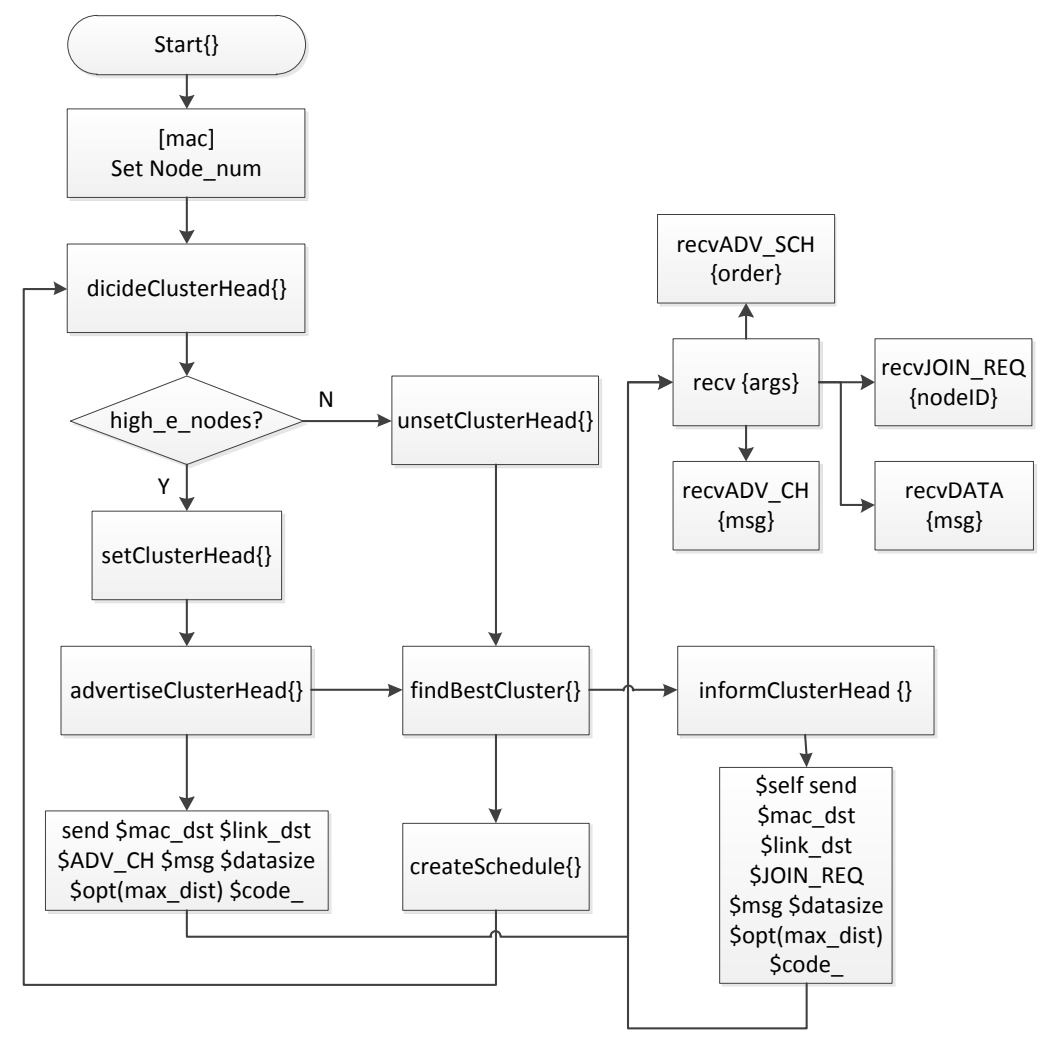

Fig. 2. Flow chart of the cluster formation process of the simplified LEACH for a fixed cluster head function 


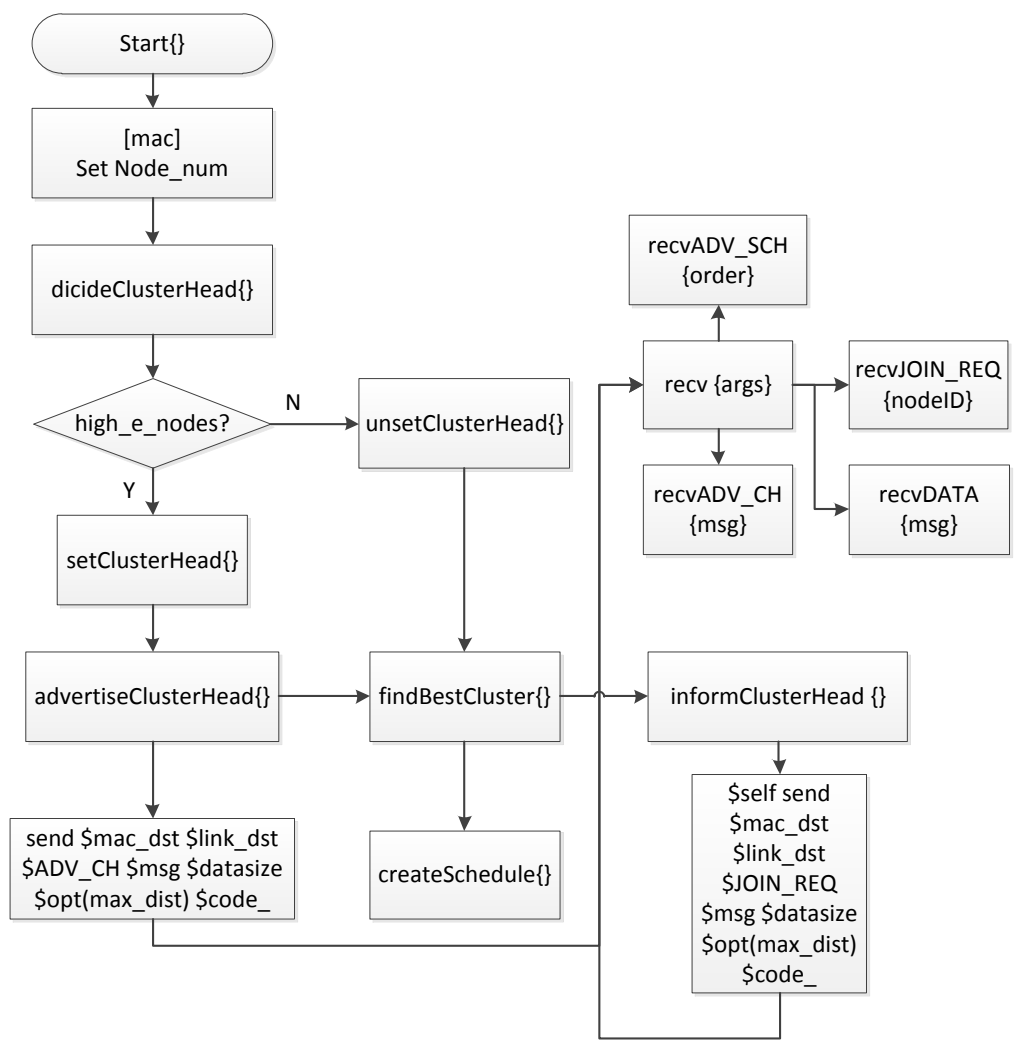

Fig. 3. Flow chart of the cluster formation process of FiCHS

for a simplified LEACH. Through experimentation, we observed some small improvements in performance. However, we observed that the clustering process in each round exhibited similar results and that in our specific environment single clustering in the first round is adequate for the end of the process. We then simplified the LEACH further by eliminating the re-clustering process to improve network performance. This configuration is called FiCHS. Fig. 3 shows the FiCHS set-up phase, where we updated the LEACH 'decideClusterHead' function so that preselected cluster head nodes that are assigned high energy broadcast advertising messages. Then, cluster heads create a TDMA schedule once for the steady state phase where data frames are transmitted to the base station. As shown in Fig. 3, the algorithm does not perform clustering and TDMA schedule creation any longer.

The set-up phase for the suggested protocol is stated below.

After assigning a mac layer address, the set-up phase enters the cluster head selection process. If a node is relevant to the retention of the highest energy level, it is selected as a cluster head and is noticed by the surrounding nodes. Otherwise, it will find a best cluster head and perform data transmission after being scheduled by the cluster head. On each periodic round, differently from FiCHS, the existing and simplified LEACH both repeats cluster head selection and performs rescheduling.

Each node simply sends data to the base station through the cluster head, just as in static clus- 
Table 1. Characteristics of each protocol

\begin{tabular}{l|l|l|l}
\hline \multicolumn{1}{c|}{ Protocol } & \multicolumn{1}{c|}{ LEACH } & \multicolumn{1}{c}{ Static clustering } & \multicolumn{1}{c}{ FiCHS } \\
\hline Algorithm & Cluster formation & $\begin{array}{l}\text { Centralized cluster for- } \\
\text { mation }\end{array}$ & Cluster formation \\
\hline Cluster head & $\begin{array}{l}\text { Election by algorithm in } \\
\text { every rounds }\end{array}$ & $\begin{array}{l}\text { Election by algorithm at } \\
\text { once }\end{array}$ & Fixed by manual operation \\
\hline $\begin{array}{l}\text { Extra H/W } \\
\text { cost }\end{array}$ & No & GPS & $\begin{array}{l}\text { High energy supply module for cluster } \\
\text { head }\end{array}$ \\
\hline
\end{tabular}

tering [2].

FiCHS does not require each node to have GPS in order to send location information for each node directly to the base station, as in static clustering [2]. This adds further effectiveness in terms of energy and cost. The characteristics for each protocol are summarized in Table 1.

\section{REsULts}

\subsection{Simulation Environments}

In order to perform simulations under the same conditions, we set simulation parameters for the size of the network, BS location, simulation time, power consumption, throughput, and number of clusters, as shown in Table 2. Each parameter value is the same as in [2] but the values for the 'base station', 'desired number of clusters', 'eq_energy', and 'high_e_nodes' parameters are set differently to take into account our specific environment.

Table 2. Simulation parameter values

\begin{tabular}{c|c}
\hline Parameter & Value \\
\hline Network gird & $(0,0) \times(100,100)$ \\
\hline Base station & $(45,45)$ \\
\hline Simulation time (seconds) & 3,600 \\
\hline$E_{\text {elec }}$ & $50 \mathrm{~nJ} / \mathrm{bit}$ \\
\hline$\in_{\text {friss-amp }}$ & $10 \mathrm{pJ} / \mathrm{bit} / \mathrm{m} 2$ \\
\hline$\in_{\text {two-ray-amp }}$ & $0.0013 \mathrm{pJ} / \mathrm{bit} / \mathrm{m} 4$ \\
\hline Bitrate & $1 \mathrm{Mbps}$ \\
\hline Desired number of clusters & 12 \\
\hline eq_energy & 0 \\
\hline high_e_nodes & 111418333641486366818488 \\
\hline
\end{tabular}

\subsection{Network Topology}

The network topology is assumed to be a mesh, as shown in Fig. 4, in consideration of the actual facility of underground power lines and maintenance access hatch locations. We reflect the distribution map for underground placement in Fig.1, to place substations (BS: red dot) and cluster heads to act as a relays with high energy (CH: orange dot), as shown in Fig. 4. 


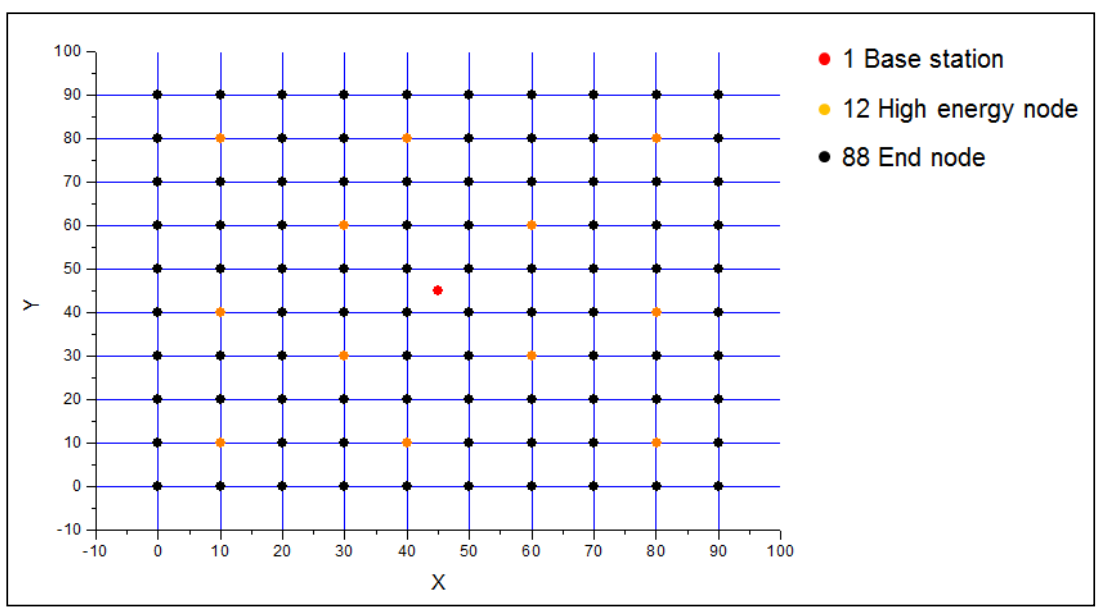

Fig. 4. 100-node mesh topology simulation map (BS: 45, 45)

\subsection{Simulation Results}

Our simulation was performed using an ns-2 network simulator with a LEACH extension [7] and implementation [8]. We compared four approaches, LEACH, LEACH-C, a simplified LEACH, and FiCHS through simulation. Fig. 5 represents the number of live nodes for each approach as time passes by in the simulation. Fig. 6 shows the total energy consumption of live nodes. Fig. 7 shows the total data received at the base station. The proposed protocol with a fixed desirable number of cluster heads sends more data to the base station than LEACH and simplified LEACH, as the sensor network lifetime for the proposed protocol is longer.

Fig. 8 compares the data received at the base station by energy. Fig. 9 compares the data received at the base station by time. From the figures, it can be seen that the proposed protocol performance is better than that of LEACH when the $\mathrm{CH}$ had high energy and a manually fixed

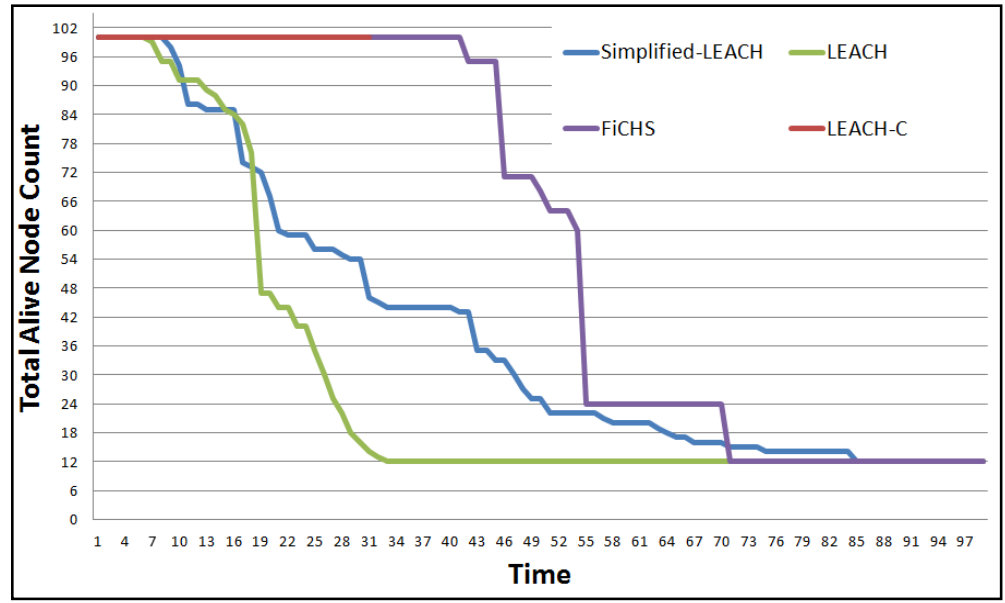

Fig. 5. Number of nodes that were alive during the simulation time 


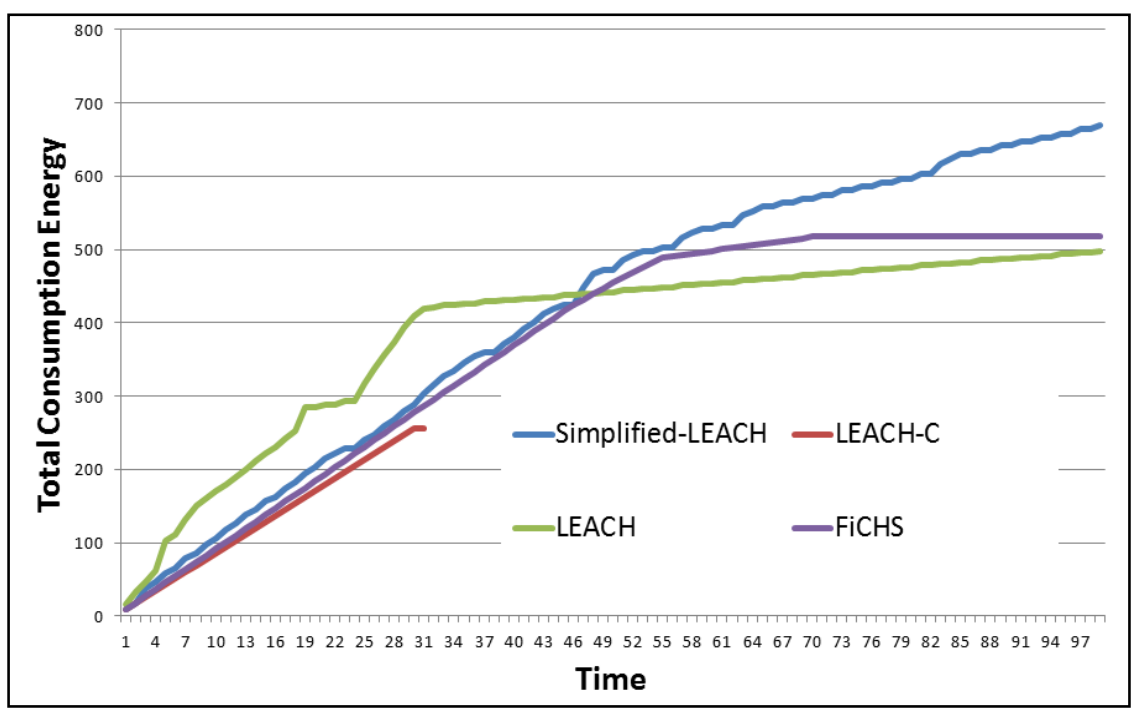

Fig. 6. Total amount of energy consumption by the nodes during the simulation time

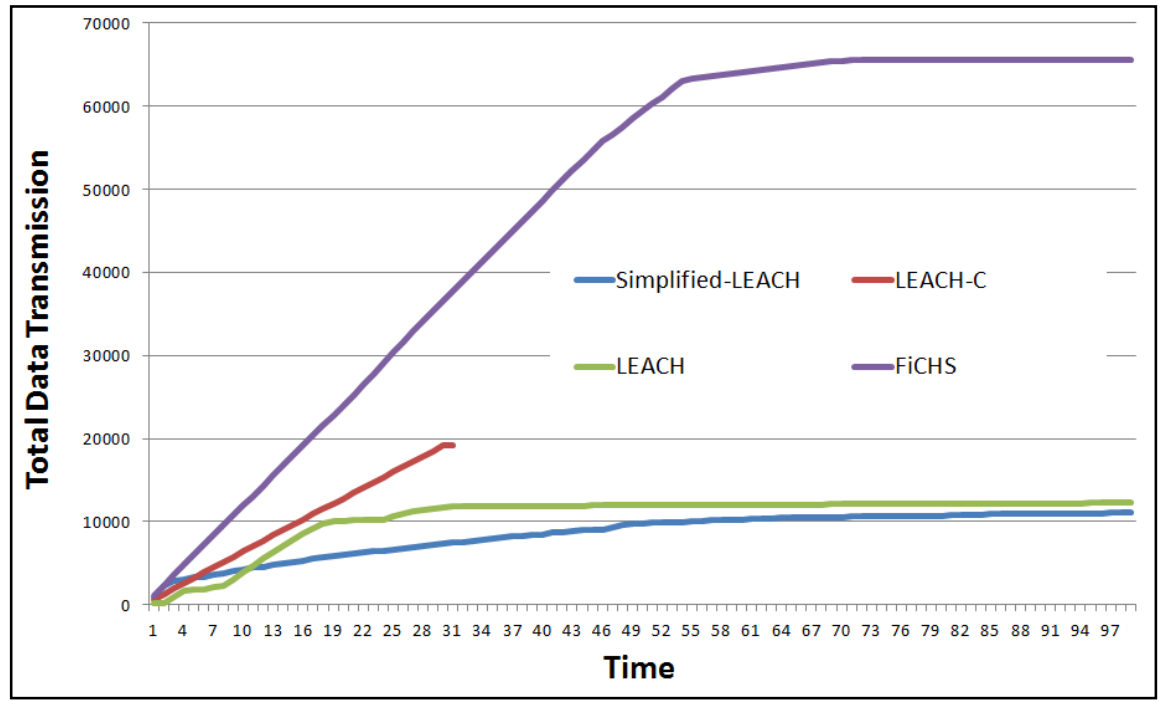

Fig. 7. Total amount of data received at the base station during the simulation time 


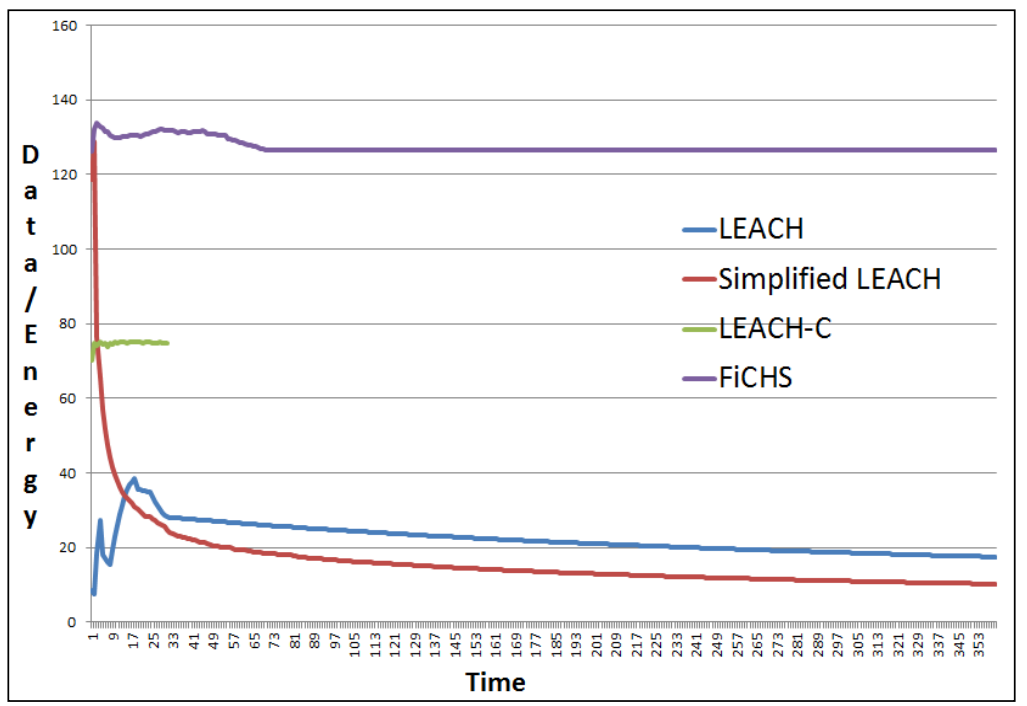

Fig. 8. Comparison of data received at the base station per energy

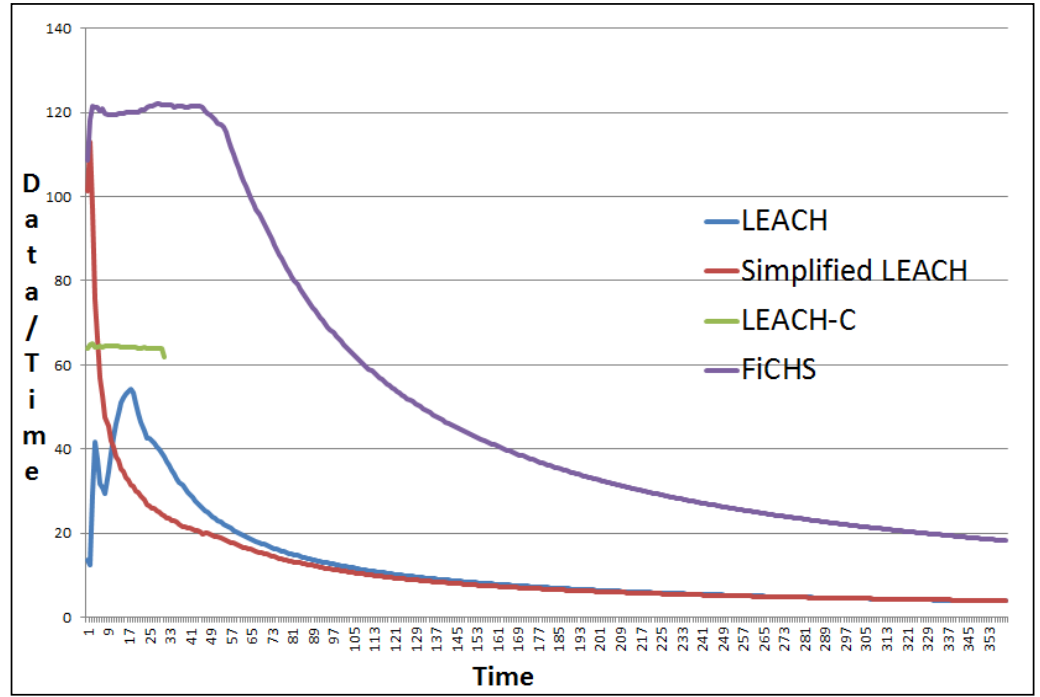

Fig. 9. Comparison of the data received at the base station per time

location. The energy level of the cluster heads in fixed locations thus plays an important role in the overall performance of energy-constrained sensor networks. LEACH-C stops after several rounds, as it is below the required number of desired clusters.

Table 3 shows the average values of each protocol. These clearly show the performance of the proposed protocol in fixed node formation and cluster heads. 
Table 3. The average of each protocol

\begin{tabular}{c|c|c|c|c}
\hline Protocol & LEACH & LEACH-C & Simplified LEACH & FiCHS \\
\hline Data/Energy & 22.24 & 74.71 & 16.44 & 127.19 \\
\hline Data/Time & 11.99 & 64.15 & 11.22 & 52.37 \\
\hline Data/Node & 138.36 & 191.71 & 142.03 & 655.74 \\
\hline
\end{tabular}

\section{CONCLUSION}

FiCHS is compared with three other protocols, LEACH, LEACH-C, and a simplified LEACH, based on an ns-2 simulation. FiCHS exhibits better performance than the other protocols. As a hierarchical routing protocol for underground power distribution line surveillance in urban areas, FiCHS has shown that $\mathrm{CH}$-driven cluster formations are desirable. The proposed protocol was also observed to enable cost cutting and a high transmission rate since the protocol does not require location information, unlike other protocols.

The proposed protocol does not yet guarantee the security function and implementation for actual surveillance. Further studies must be carried out to resolve these problems.

\section{REFERENCES}

[1] Heinzelman W. and Chandrakasan A., Balakrishnan H. "Energy-Efficient Communication Protocol for Wireless Microsensor Networks". In: Proceedings of the 3rd Annual Hawaii Int'1 Conference on System Sciences. Maui: IEEE Computer Society, 2000, 3005-3014.

[2] Heinzelman W. and Chandrakasan A. "An Application Specific Protocol Architectures for wireless Microsensor Networks". [Ph.D. Thesis]. Boston: Massachusetts Institute of Technology, 2000.

[3] Hong Li, Xu Shunjie, Li Shurong, Zou Weixia and Zhou Zheng, "Novel Method for Optimal Number of Cluster Heads in LEACH”, 2009 WASE International Conference on Information Engineering, 2009, 302-309.

[4] “The MIT uAMPS ns Code Extensions Version 1.0,” http://www-mtl.mit.edu/researchgroups/ icsystems/uamps/research/leach/leach_doc.pdf

[5] Jason A. Pamplin, "NS2 Leach Implementation," http://www.internetworkflow.com/resources/ ns2leach.pdf.

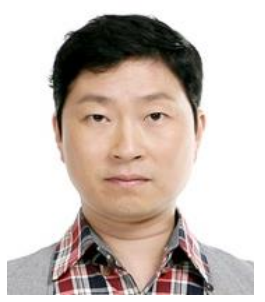

\section{Hoo-Rock Lee}

He received the BS degrees in Information Engineering and Computer Science from Joong-bu Univ. in 1995 and 2002 and the MS degrees in Computer Engineering from Chung-nam Univ. in 2002 and 2004. Since 2002, he stayed in Korea Institute of Machinery and Materials to develop the wireless communication applications. And now he is undertaking a doctorate course as a member of the digital system lab at Chung-nam Univ. His research interests include in the intelligent surveillance of industrial plant. 


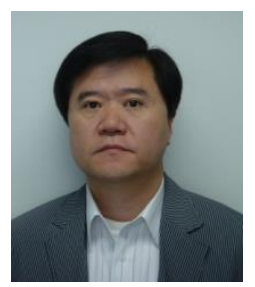

\section{Kyung-Yul Chung}

He received a Ph.D. degree in System Engineering from Korea-maritime Univ. in 1997. He has been a principal researcher at Korea Institute of Machinery and Materials since 1987. His research interests are in the Power Plant Engineering, Electrical Facility Diagnosis, Environment Processing and Maritime Engineering.

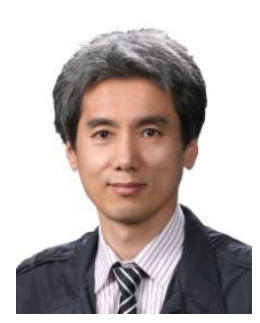

\section{Kyoung-Son Jhang}

He received his Ph.D. degree in Computer Engineering from Seoul National Univ. in 1995. He joined the faculty members of Dept. of Computer Engineering in Chungnam National Univ. from 2001. His current research interests include image processing and parallel programming with CUDA. 